\title{
Suture Anchor Repair With Fully Threaded Knotless Anchors for Quadriceps Tendon Rupture Resulted in Favorable Outcomes After 2 Years
}

\author{
Ryo Sasaki, M.D., Masaki Nagashima, M.D., Ph.D., Toshiro Otani, M.D., Ph.D., \\ Yoshifumi Okada, M.D., Shinsuke Aida, M.D., Ph.D., Kenichiro Takeshima, M.D., Ph.D., \\ and Ken Ishii, M.D., Ph.D.
}

\begin{abstract}
Purpose: To investigate clinical outcomes over 2 years in cases of quadriceps tendon rupture (QTR) that were surgically treated using fully threaded knotless anchors. Methods: A total of four knees in four male patients with QTR repaired with fully threaded knotless anchors at our hospital from November 2017 to January 2019 were enrolled. Mean patient age at surgery was 65.3 years (range: 61-70 years). Intraoperatively, stability of the sutured site was confirmed by knee flexion to $90^{\circ}$. Full weight walking with the orthosis in extension was commenced on the seventh postoperative day. Surgical findings, pathologies of the ruptured quadriceps tendons, and postoperative clinical outcomes were evaluated in all patients. Results: The QTR was complete in three cases and partial in one. Average surgical duration was 58.5 (range: 49-74) minutes. Pathological evaluation revealed hyaline degeneration with granulation of the quadriceps tendon in two cases. No complications, such as infection and rerupture, occurred. Magnetic resonance imaging performed 1 year postoperatively confirmed complete healing of the repaired tendon. The mean follow-up period was 35.5 months (range: $24-46$ months). None of the patients had extension lag of the knee, and mean Lysholm score and range of flexion were 95.3 (range: 85-100) and $141.3^{\circ}$ (range: $140-145^{\circ}$ ), respectively, at the final follow-up. Conclusions: Clinical outcomes were favorable in all cases, including two cases with pathological degenerative changes. Suture anchor repair with fully threaded knotless anchors can be considered a minimally invasive and effective method for QTR, with sufficient strength to allow early rehabilitation.
\end{abstract}

\section{Introduction}

Q uadriceps tendon rupture (QTR) is a rare injury that is sometimes seen in elderly patients and those with renal failure and diabetes. ${ }^{1}$ QTR is sometimes difficult to diagnose, and patients occasionally

Department of Orthopaedic Surgery, School of Medicine, International University of Health and Welfare, Chiba, Japan (R.S., M.N., K.T., K.I.); Department of Orthopaedic Surgery, International University of Health and Welfare, Mita Hospital, Tokyo, Japan (R.S., M.N., Y.O., K.T., K.I.); Department of Orthopaedic Surgery, International University of Health and Welfare, Ichikawa Hospital, Chiba, Japan (T.O.); Department of Pathology, International University of Health and Welfare, Mita Hospital, Tokyo, Japan (S.A.); and Department of Orthopaedic Surgery, International University of Health and Welfare, Narita Hospital, Chiba, Japan (R.S., M.N., K.T., K.I.).

Received June 2, 2021; accepted September 20, 2021.

Address correspondence to Masaki Nagashima, M.D., Ph.D., Department of Orthopaedic Surgery, International University of Health and Welfare, Mita Hospital, 1-4-3, Mita, Minato-ku, Tokyo 108-8329, Japan. E-mail: masakin@iuhw.ac.jp

(C) 2021 THE AUTHORS. Published by Elsevier Inc. on behalf of the Arthroscopy Association of North America. This is an open access article under the CC BY-NC-ND license (http://creativecommons.org/licenses/by-nc-nd/4.0/).

2666-061X/21776

https://doi.org/10.1016/j.asmr.2021.09.012 come to the hospital in the chronic condition. ${ }^{2}$ Patients with QTR have difficulty walking with active extension of the knee and have significant functional disability. Furthermore, QTR requires surgical repair to avoid the poor outcomes previously reported in cases of neglected and chronic rupture. ${ }^{3,4}$ Although several surgical techniques have been reported for repair of QTR, such as pull-out repair and augmentation, it remains unclear which method is the most effective. Suture anchor repair is reported to be minimally invasive and simple in cases in which the rupture site is near the patella, enabling strong suturing, equivalent to pull-out repair. ${ }^{5}$ The fully threaded knotless anchor (SwiveLock; Arthrex, Naples, FL) that was applied in this study has been approved as a novel anchor system. The fully threaded knotless anchor consists of two components. The distal component can hold several sutures or strength tape, and the proximal component can attach closely to bone. Hence, the fully threaded knotless anchor has been reported to have the highest load to failure among the suture anchors. ${ }^{6}$ Therefore, although favorable postoperative results might be expected when the fully threaded knotless anchor is used for repairing 

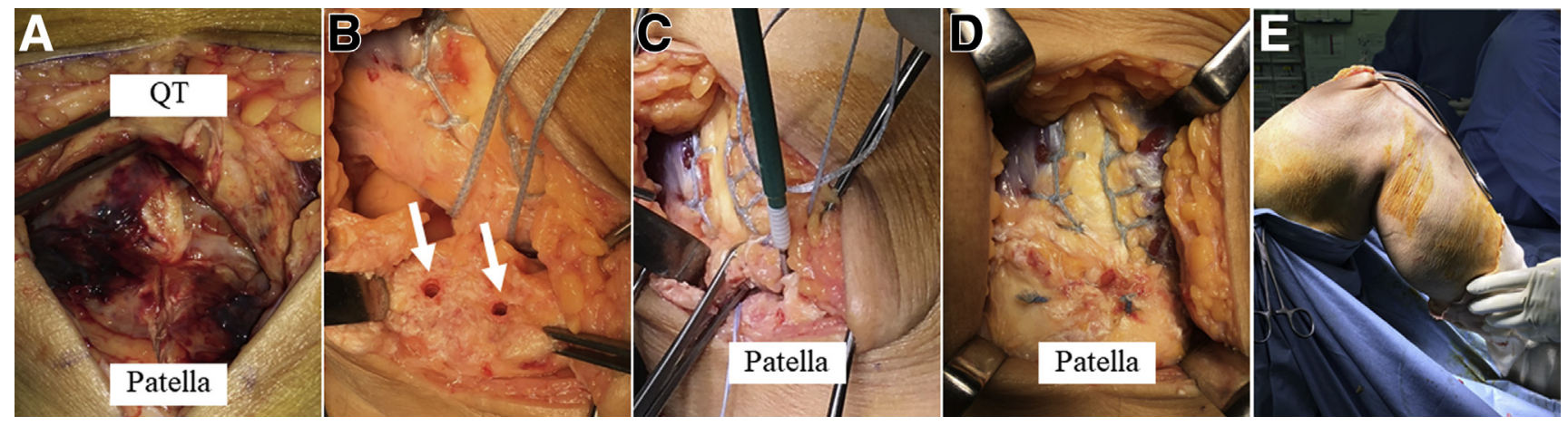

Fig 1. Surgical procedure. (A) An anterior longitudinal incision almost $8 \mathrm{~cm}$ in length exposed the distal end of the ruptured quadriceps tendon (QT) and the proximal pole of the patella. (B) The strength tape was passed through the distal margin of the ruptured quadriceps tendon using the Krackow stitch, and two osseous holes were drilled in the proximal part of the patella (white arrows). (C) The strength tape was threaded through the distal component of the 4.75-mm SwiveLock and anchored into each osseous hole. The strings attached to the SwiveLock were also used for suturing. (D) Any additional tears were sutured appropriately with absorbable surgical sutures. (E) The suture site was checked for instability when the knee was flexed to $90^{\circ}$.

tendon rupture, to the best of our knowledge, there have been no previous reports showing the clinical outcomes of quadriceps tendon repair using a fully threaded knotless anchor.

The purpose of this study was to investigate clinical outcomes over 2 years in cases of QTR that were surgically treated using fully threaded knotless anchors. Our hypothesis was that suture anchor repair with fully threaded knotless anchors for QTR leads to a favorable clinical outcome.

\section{Materials and Methods}

\section{Subjects}

Patients with QTR repaired using fully threaded knotless anchors at our hospital from November 2017 to January 2019 were identified. Preoperative demographics of the patients, including body weight, body mass index, medical history, mechanism of injury, and time from injury to surgery were retrospectively reviewed. For all of the patients, tenderness, swelling and indentation at the site of injury in the quadriceps tendon were confirmed preoperatively, and radiographs showed anterior tilting of the patella. Rupture of the quadriceps tendon was observed preoperatively by magnetic resonance imaging (MRI). This retrospective study was approved by our institutional review board (file no. 5-16-55), and written informed consent was obtained from all patients.

\section{Availability of Data and Materials}

The datasets of the present study are available from the corresponding author upon reasonable request.

\section{Surgical Procedure}

QTR repairs were performed under general anesthesia and tourniquet use. Suture anchor repair with the SwiveLock was performed when the ruptured portion was within $2 \mathrm{~cm}$ from the proximal end of the patella (Fig 1). (A) An anterior longitudinal incision almost $8 \mathrm{~cm}$ in length exposed the distal end of the ruptured quadriceps tendon and the proximal pole of the patella. (B) A piece of the ruptured tendon tissue was collected for pathological examination. The

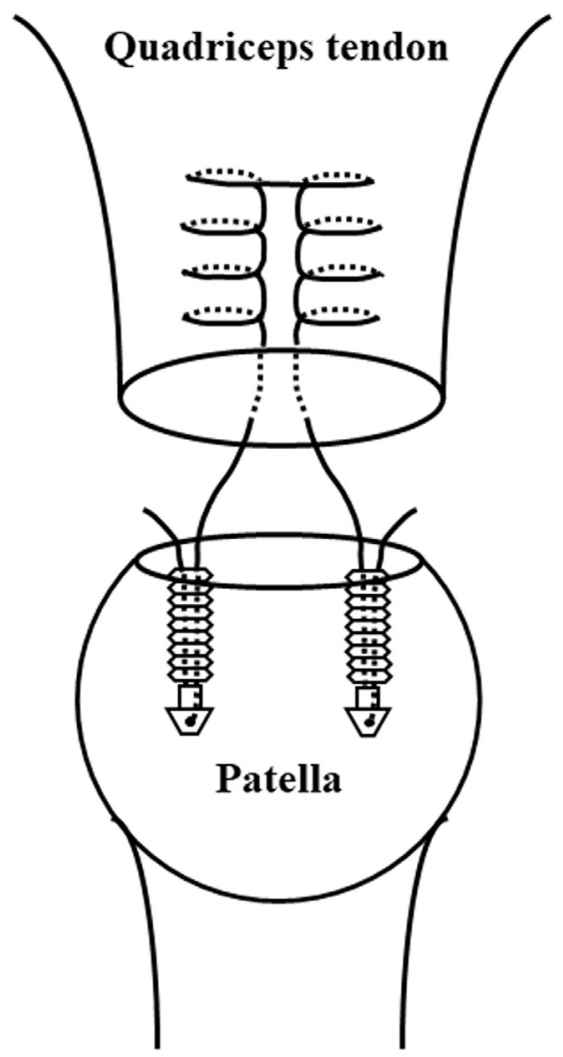

Fig 2. Schematic diagram of suture anchor repair with fully threaded knotless anchors. The strength tape was passed through the distal margin of the ruptured quadriceps tendon using the Krackow stitch. Two osseous holes were drilled in the proximal part of the patella, and the strength tape was anchored into the osseous holes using a fully threaded knotless anchor. 
Table 1. Demographic Data of the Patients

\begin{tabular}{lccccclcr}
\hline & & \multicolumn{5}{c}{ Body } & & Time from Injury \\
Patient No. & Age $(\mathrm{yr})$ & Sex & Side & Weight $(\mathrm{kg})$ & BMI $\left(\mathrm{kg} / \mathrm{m}^{2}\right)$ & Mechanism of Injury & \multicolumn{2}{c}{$\begin{array}{r}\text { Comorbidities } \\
\text { to Surgery }(\text { days })\end{array}$} \\
\hline 1 & 68 & Male & Right & 90 & 30.1 & Fell while walking & Hyperlipidemia, Diabetes \\
2 & 62 & Male & Right & 73 & 25.1 & Fell during golf & Hypertension & 4 \\
3 & 61 & Male & Right & 65 & 21.0 & Fell on the stairs & Hypertension, Asthma \\
4 & 70 & Male & Right & 93 & 32.2 & Fell from height & Diabetes & 3 \\
\hline
\end{tabular}

BMI, body mass index.

strength tape was passed through the distal margin of the ruptured quadriceps tendon using the Krackow stitch, ${ }^{7}$ and two osseous holes were drilled in the proximal part of the patella. $(C)$ The strength tape was threaded through the distal component of the $4.75-\mathrm{mm}$ SwiveLock and anchored into each osseous hole. The schematic diagram of suture anchor repair with fully threaded knotless anchors was shown in Fig 2. (D) The strings attached to the SwiveLock were also used for suturing. Any additional tears were sutured appropriately with absorbable surgical sutures. (E) The suture site was checked for instability when the knee was flexed to $90^{\circ}$. The surgical plan was to perform augmentation using an artificial ligament in case instability of the suture site was recognized.

\section{Postoperative Care}

Postoperatively, the knee was immobilized with a brace for 2 weeks. Full weight-bearing with the orthosis in extension was authorized on the seventh postoperative day. After the knee immobilization, range of motion exercises and muscle strengthening were commenced. Initially, the flexion angle was limited to $90^{\circ}$ for 2 weeks, following which full flexion was allowed. The hinged knee brace was used for 3 months. Although none of the patients received pharmacological prophylaxis for deep vein thrombosis (DVT), intermittent pneumatic compression was used for 1 day, and compression stockings were used for 2 weeks after the surgery.

\section{Surgical Findings and Clinical Outcomes}

To assess the surgical findings, the site and condition of tendon rupture, operative time, and pathology of the ruptured quadriceps tendon were reviewed, and complications, such as infection, implant dislocation, DVT, and rerupture were recorded. As clinical outcomes, postoperative Lysholm score, range of flexion, and extension lag were examined at the final visit.

\section{Results}

A total of four knees in four patients with QTR were enrolled in the present study. In the repair surgeries, fully threaded knotless anchors were used at our hospital from November 2017 to January 2019. All four patients were male, and their mean age was 65.3 years. Preoperative demographics of the patients, including body weight, body mass index, medical history, mechanism of injury, and time from injury to surgery were retrospectively reviewed and are shown in Table 1 . Intraoperative findings showed that all QTRs occurred within $2 \mathrm{~cm}$ proximal to the superior pole of the patella. Three cases had complete rupture of the quadriceps tendon, and one case involved rupture of the rectus femoris and vastus medialis tendons. There was no instability of the tendon at $90^{\circ}$ knee flexion after repair of the QTRs with the suture anchor in any of the cases, and hence, artificial ligament augmentation was not performed. The average operative time was 58.5 (range: 49-74) minutes. Pathological evaluation revealed hyaline and mucoid degeneration with granulation of the quadriceps tendon in two cases. No complications occurred in any of the cases. MRI performed 1 year after the surgery confirmed complete healing of the repaired tendon. The mean follow-up interval was 35.5 months (range: 24-46 months). Postoperatively, none of the patients had extension lag of the knee, and mean Lysholm score and range of flexion at the last follow-up were 95.3 (range: 85-100) and $141.3^{\circ}$ (range: $140-145^{\circ}$ ), respectively (Table 2 ).

Table 2. Surgical Findings and Postoperative Clinical Outcomes

\begin{tabular}{|c|c|c|c|c|c|c|c|}
\hline $\begin{array}{l}\text { Patient } \\
\text { Number }\end{array}$ & $\begin{array}{l}\text { Operative } \\
\text { Time (min) }\end{array}$ & $\begin{array}{c}\text { Partial vs } \\
\text { Complete Rupture }\end{array}$ & $\begin{array}{c}\text { Pathological } \\
\text { Tendon } \\
\text { Degeneration }\end{array}$ & $\begin{array}{c}\text { Time to Follow-Up } \\
\text { (Months) }\end{array}$ & $\begin{array}{c}\text { Lysholm } \\
\text { Score (Points) }\end{array}$ & $\begin{array}{c}\text { Range of } \\
\text { Flexion (Degrees) }\end{array}$ & Extension Lag \\
\hline 1 & 60 & Complete & - & 46 & 100 & 140 & - \\
\hline 3 & 49 & Partial & + & 36 & 100 & 140 & - \\
\hline 4 & 51 & Complete & + & 24 & 85 & 140 & - \\
\hline
\end{tabular}




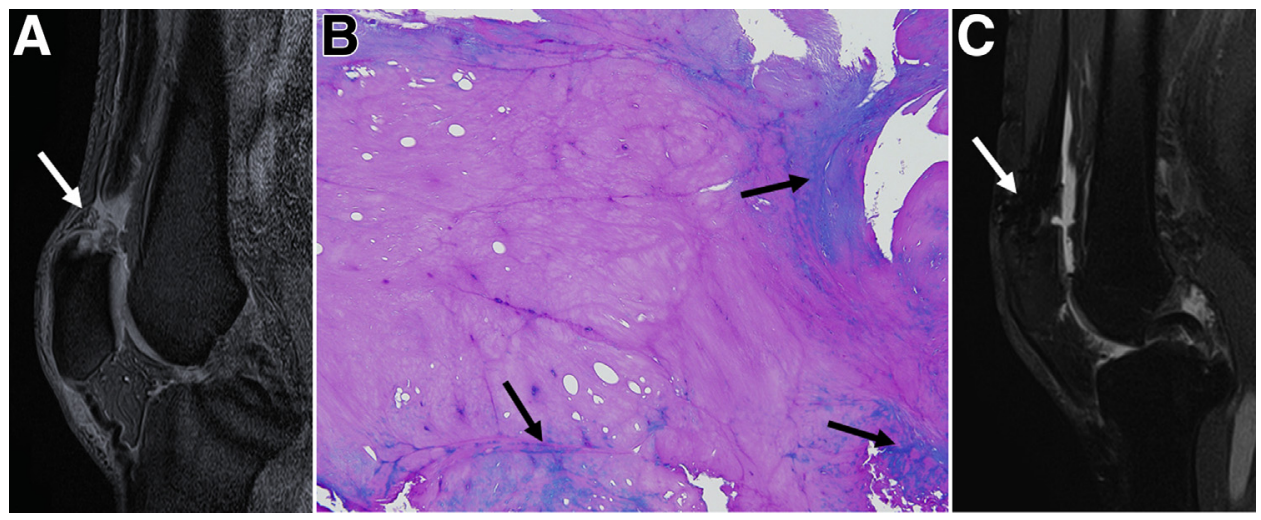

Fig 3. Magnetic resonance and histopathological findings in a representative case (Case 3 ). (A) Preoperative magnetic resonance imaging (MRI) of the affected knee. A sagittal T2 star-weighted image showed rupture of the quadriceps tendon (white arrow). (B) Pathological findings of the ruptured quadriceps tendon (Alcian blue/periodic acid-Schiff stain, $10 \times)$. Overall hyaline degeneration and some bluestained mucoid degeneration (black arrows) were seen. (C) Postoperative MRI of the affected knee performed 1 year after the surgery. A sagittal fat-suppressed T2-weighted image showed complete healing of the repaired tendon (white arrow).

\section{Case presentation (Case 3)}

A 70-year-old man visited our hospital complaining of right knee pain and difficulty in walking. He had injured his right knee in a fall 24 days before his first visit to our clinic. He had a history of diabetes and was under treatment with insulin. Physical examination revealed swelling of the right knee and an indentation on the proximal side of the patella. He was unable to extend his right knee by himself, and MRI showed quadriceps tendon rupture within $2 \mathrm{~cm}$ proximal to the upper end of the patella (Fig 3A). Surgery was performed 32 days after the injury. Intraoperative evaluation showed that the quadriceps tendon was completely ruptured, and suture anchor with SwiveLock was performed. On pathological analysis, hyaline and mucoid degeneration with granulation of his ruptured tendon was recognized (Fig 3B). His postoperative course was good and uneventful. MRI confirmed complete healing of the repaired tendon at 1 year after surgery (Fig $3 \mathrm{C}$ ). The range of flexion at the last follow-up was $140^{\circ}$, and there was no extension lag of the knee (Fig 4).

\section{Discussion}

In the current study, suture anchor repair with fully threaded knotless anchors was applied in four cases with QTRs, and good clinical results over a follow-up period of more than 2 years were obtained, even in cases in which degeneration of the tendon was seen on pathological analysis. No instability was observed even at $90^{\circ}$ of flexion intraoperatively, and despite the simple procedure, sufficient suture strength was obtained to allow early rehabilitation. The results of the present study demonstrated that the suture anchor repair was an effective method to treat QTR and supported the hypothesis that suture anchor repair with fully threaded knotless anchors for QTR leads to a favorable clinical outcome.

To date, several studies about the epidemiology of QTR have been reported. These studies reported that QTRs are rare injuries with a reported incidence of 1.37 cases per $100,000,{ }^{8}$ and that elderly and obese people are more susceptible to this trauma. ${ }^{9,10}$ In addition, medical histories of comorbidities, such as renal failure, rheumatoid arthritis, systemic lupus erythematosus, oral steroids and hyperparathyroidism are risk factors for QTR. ${ }^{1}$ The QTR developed secondary to a fall in most patients, and the tendon rupture sites were close to the proximal attachment of the tendon to the patella in most cases. ${ }^{11}$ A previous study reported that the ruptured tendon usually demonstrated pathological changes, such as hypoxic degeneration, mucoid degeneration, endolipomatosis, and other types of degeneration, ${ }^{12}$ suggesting that degenerative changes in the tissue of the ruptured tendon should be taken into account when treating QTR.

Minimally invasive and strong repair techniques are desirable for the treatment of QTR. Such procedures allow early rehabilitation, which reduces patient burden (e.g., hospitalization and immobilization periods), and reduce complications such as DVT. Combination of reattachment of the tendon through bone tunnels and tendon augmentation was previously reported to be strong enough to safely permit early mobility, weight bearing and brace-free ambulation. ${ }^{13}$ Furthermore, it has been reported that early rehabilitation with a strong repair leads to expedited return to work, without increasing the incidence of re-rupture. ${ }^{14}$ 
Fig 4. Postoperative photographs of the patient's affected knee in extension and flexion. The range of flexion at the last follow-up was $140^{\circ}(\mathrm{A})$, and there was no extension lag of the knee (B).
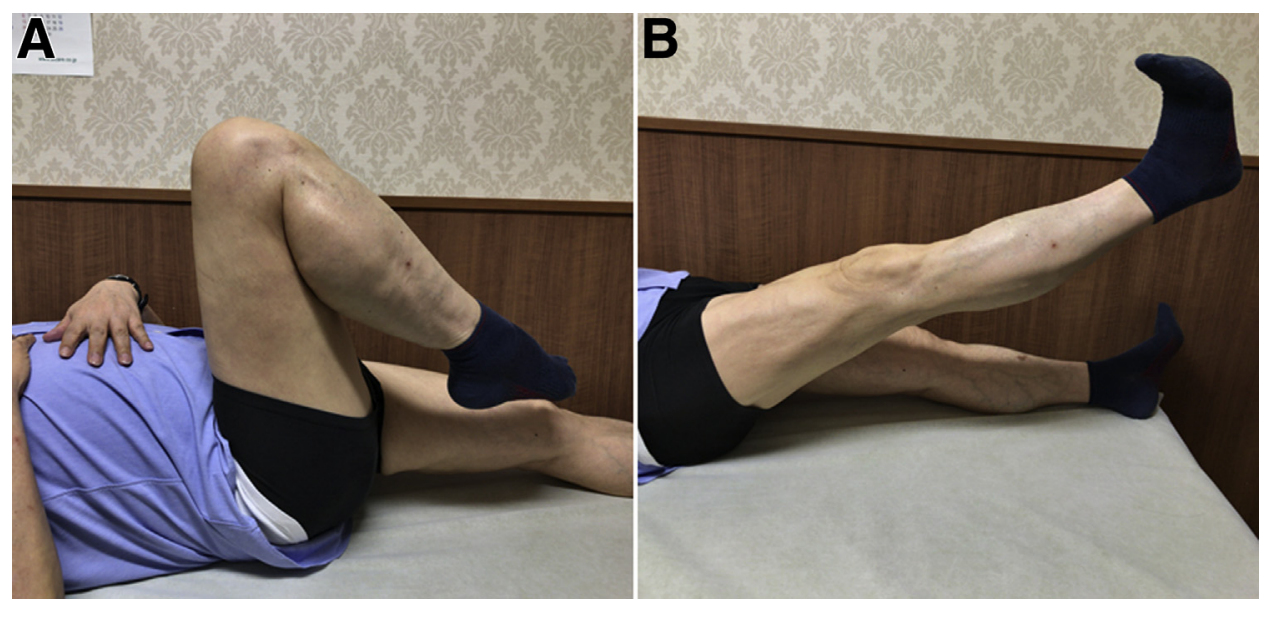

QTR is more common in elderly patients, and advanced age is a risk factor for DVT. ${ }^{15}$ Since serious venous thrombosis has been reported after the surgical treatment of QTR, ${ }^{9}$ adequate DVT prevention, including early rehabilitation, should be considered. Traditionally, QTR was typically treated by reattaching the ruptured tendons by end-to-end suturing of the torn tendon. This method requires sufficient length of the distal part of the ruptured tendon. However, most QTRs occur within $2 \mathrm{~cm}$ proximal to the patella. In addition, several reports have described inadequate strength of the repaired tendon. ${ }^{16}$ Favorable surgical outcomes have also been described with the previously reported technique of reattaching the tendon through bone tunnels with or without tendon or hardware augmentation, ${ }^{13}$ although these methods were shown to be invasive and complicated. The previously described technique also requires making a skin incision from the proximal to the distal part of the patella to make drill holes for bone tunnels, while augmentation methods need a long skin incision from the distal end of the patella to the end of the proximal part of the ruptured tendon. In contrast, suture anchor repair requires only a small incision from the superior pole of the patella to the area of the tendon rupture, and is less invasive, while still permitting strong repair of the ruptured tendon, ${ }^{17}$ regardless of the margin available for sewing. In fact, a recent cadaveric study reported that there was no statistically significant difference in ultimate load to failure between suture anchor repair and reattaching the tendon through bone tunnels. ${ }^{5,18-20}$

A previous study reported that fully threaded knotless anchors showed the highest failure strengths among other types of suture anchors ${ }^{6}$. Furthermore, a case of suture anchor repair with fully threaded knotless has been reported in a technical note article as an effective method for QTR. ${ }^{21}$ Thus, it was considered that suture anchor repair with fully threaded knotless anchors had the potential to increase the strength of the repaired tendon. However, the clinical outcomes of quadriceps tendon repair with fully threaded knotless anchors have so far remained unknown.

This case series involved two cases with degenerative changes of the quadriceps tendon and one case in which surgery was performed in the chronic phase, defined as 3 weeks after the time of injury. ${ }^{22}$ As mentioned earlier, the quadriceps tendon showed degeneration in many previously reported cases of QTR, especially in chronic cases, making it difficult to achieve a strong repair. However, in this study, it was shown that suture anchor repair with a fully threaded knotless anchor was very effective, even for QTRs with degeneration.

\section{Limitations}

This study is not without limitations. First, it included a very small number of patients. Second, as this was a case series, there were no control cases.

\section{Conclusion}

Clinical outcomes were favorable in all four cases, including two cases with pathological degenerative changes. Suture anchor repair with fully threaded knotless anchors can be considered a minimally invasive and effective method for QTR, with sufficient strength to allow early rehabilitation.

\section{References}

1. Kim BS, Kim YW, Song EK, Seon JK, Kang KD, Kim HN. Simultaneous bilateral quadriceps tendon rupture in a patient with chronic renal failure. Knee Surg Relat Res 2012;24:56-59.

2. Neubauer T, Wagner M, Potschka T, Riedl M. Bilateral, simultaneous rupture of the quadriceps tendon: a diagnostic pitfall? Report of three cases and meta-analysis of the literature. Knee Surg Sports Traumatol Arthrosc 2007;15: 43-53. 
3. Scuderi C. Ruptures of the quadriceps tendon; study of twenty tendon ruptures. Am J Surg 1958;95:626-634.

4. Siwek CW, Rao JP. Ruptures of the extensor mechanism of the knee joint. J Bone Joint Surg Am 1981;63:932-937.

5. Sherman SL, Copeland ME, Milles JL, Flood DA, Pfeiffer FM. Biomechanical evaluation of suture anchor versus transosseous tunnel quadriceps tendon repair techniques. Arthroscopy 2016;32:1117-1124.

6. Barber FA, Herbert MA, Beavis RC, Barrera Oro F. Suture anchor materials, eyelets, and designs: Update 2008. Arthroscopy 2008;24:859-867.

7. Labib SA, Hoffler CE 2nd, Shah JN, Rolf RH, Tingan A. The gift box open Achilles tendon repair method: A retrospective clinical series. J Foot Ankle Surg 2016;55:39-44.

8. Ciriello V, Gudipati S, Tosounidis T, Soucacos PN, Giannoudis PV. Clinical outcomes after repair of quadriceps tendon rupture: A systematic review. Injury 2012;43: 1931-1938.

9. Hansen L, Larsen S, Laulund T. Traumatic bilateral quadriceps tendon rupture. J Orthop Sci 2001;6:187-188.

10. Boudissa M, Roudet A, Rubens-Duval B, Chaussard C, Saragaglia D. Acute quadriceps tendon ruptures: a series of 50 knees with an average follow-up of more than 6 years. Orthop Traumatol Surg Res 2014;100:213-216.

11. Shah MK. Simultaneous bilateral quadriceps tendon rupture in renal patients. Clin Nephrol 2002;58:118-121.

12. Kannus P, Józsa L. Histopathological changes preceding spontaneous rupture of a tendon. A controlled study of 891 patients. J Bone Joint Surg Am 1991;73:1507-1525.

13. West JL, Keene JS, Kaplan LD. Early motion after quadriceps and patellar tendon repairs: Outcomes with singlesuture augmentation. Am J Sports Med 2008;36:316-323.
14. Langenhan R, Baumann M, Ricart P, et al. Postoperative functional rehabilitation after repair of quadriceps tendon ruptures: a comparison of two different protocols. Knee Surg Sports Traumatol Arthrosc 2012;20:2275-2278.

15. Nagashima M, Otani T, Takeshima K, et al. Unexpectedly high incidence of venous thromboembolism after arthroscopic anterior cruciate ligament reconstruction: Prospective, observational study. J ISAKOS 2020;5:80-82.

16. Wenzl ME, Kirchner R, Seide K, Strametz S, Jürgens C. Quadriceps tendon ruptures-is there a complete functional restitution? Injury 2004;35:922-926.

17. Lighthart WA, Cohen DA, Levine RG, Parks BG, Boucher HR. Suture anchor versus suture through tunnel fixation for quadriceps tendon rupture: A biomechanical study. Orthopedics 2008;31:441.

18. Paci JM, Pawlak A. Knotless tape suture fixation of quadriceps tendon rupture: A novel technique. Am J Orthop (Belle Mead NJ) 2018;47.

19. Massey PA, Myers M, McClary K, Brown J, Barton RS, Solitro GF. Biomechanical analysis of patellar tendon repair with knotless suture anchor tape versus transosseous suture. Orthop J Sports Med 2020;8: 2325967120954808.

20. Kindya MC, Konicek J, Rizzi A, Komatsu DE, Paci JM. Knotless suture anchor with suture tape quadriceps tendon repair is biomechanically superior to transosseous and traditional suture anchor-based repairs in a cadaveric model. Arthroscopy 2017;33:190-198.

21. Amini MH. Quadriceps tendon repair using knotless anchors and suture tape. Arthrosc Tech 2017;6:e1541-e1545.

22. Rougraff BT, Reeck CC, Essenmacher J. Complete quadriceps tendon ruptures. Orthopedics 1996;19:509-514. 